\title{
Analytical Solution for Bending Stress Intensity Factor from Reissner's Plate Theory
}

\author{
Lalitha Chattopadhyay \\ National Aerospace Laboratories, Bangalore, India \\ E-mail: lalitha@nal.res.in \\ Received December 30, 2010; revised January 27, 2011; accepted April 7, 2011
}

\begin{abstract}
Plate-type structural members are commonly used in engineering applications like aircraft, ships nuclear reactors etc. These structural members often have cracks arising from manufacture or from material defects or stress concentrations. Designing a structure against fracture in service involves consideration of strength of the structure as a function of crack size, dimension and the applied load based on principles of fracture mechanics. In most of the engineering structures the plate thickness is generally small and in these cases though the classical plate theory has provided solutions, the neglect of transverse shear deformation leads to the limitation that only two conditions can be satisfied on any boundary whereas we have three physical boundary conditions on an edge of a plate. In this paper this incompatibility is eliminated by using Reissner plate theory where the transverse shear deformation is included and three physically natural boundary conditions of vanishing bending moment, twisting moment and transverse shear stress are satisfied at a free boundary. The problem of estimating the bending stress distribution in the neighbourhood of a crack located on a single line in an elastic plate of varying thickness subjected to out-of-plane moment applied along the edges of the plate is examined. Using Reissner's plate theory and integral transform technique, the general formulae for the bending moment and twisting moment in an elastic plate containing cracks located on a single line are derived. The thickness depended solution is obtained in a closed form for the case in which there is a single crack in an infinite plate and the results are compared with those obtained from the literature.
\end{abstract}

Keywords: Reissner Plate Theory, Integral Transform, Stress Intensity Factor, Singular Integral Equation.

\section{Introduction}

In the classical theory of bending of thin plates, it is possible to satisfy stress-free conditions at an edge only in an approximate way, since only two boundary conditions may be enforced in connection with the bi-harmonic differential equation. It is the purpose of this paper to examine the crack problem by using the theory of bending of elastic plates developed by Reissner[1] in which the three physically natural boundary conditions of vanishing bending moment, twisting moment and transverse shear stress must be satisfied at a free boundary.

The present problem is concerned with the problem of an infinite plate under uniform uniaxial bending far from the crack (Figure 1). In the present work the complete solution is obtained for bending stresses in the vicinity of a crack tip in a plate taking transverse shear deformation into account through the use of Reissner's plate theory.
Using Reissner's theory and integral transform techniques, the general formulae for the bending moment,twisting moment and bending stress distribution in an elastic plate containing cracks located on a single line are derived.

The procedure employed here is to formulate the problem in terms of Reissner's equations. The stress intensity factor is obtained for the case in which there is a single crack in an infinite plate and the results are compared with those given in the literature.

The mechanical behavior near the crack tip is modeled using Reissner's plate theory in the case of an elastic plate in $[2,3]$.Effect of plate thickness on the bending stress distribution is included by Hartranft and Sih [2]. The general solution for bending stress in the vicinity of a crack tip in a plate taking shear deformation into account through the use of a sixth order plate bending theory of Reissner's theory is developed by Viswanath [3]. 


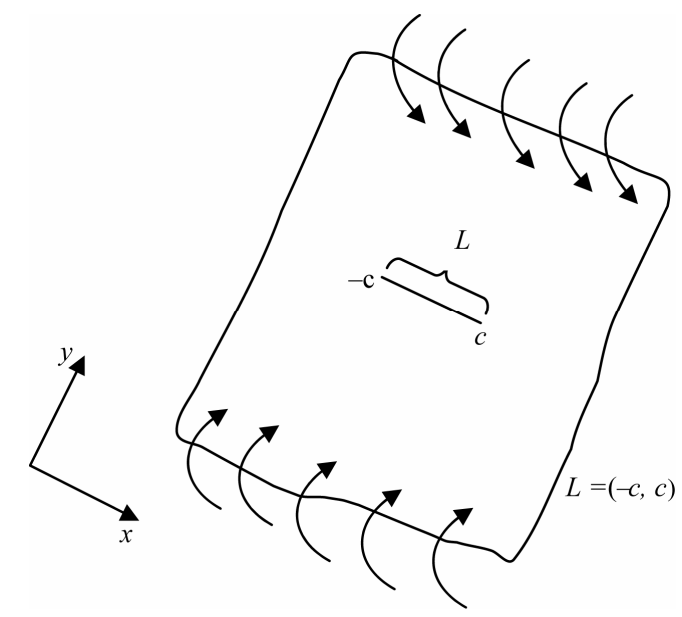

Figure 1. Plate containing a single crack and subjected to symmetric bending load.

The solution of the thin plate-bending problem was pioneered by Williams [4], who made use of the eigen function expansion technique and determined the stress distribution in the neighborhood of a crack. Sih et al. [5] applied a complex variable method for evaluating the strength of stress singularities at crack tips in plate extension and bending problems. The general solution for finite number of cracks using anisotropic elasticity is presented by Krenk[6]. Alwar and Ramachandran [7] showed that the stress intensity factor is nearly linear through the thickness for thin plates, in the absence of crack closure. Using finite element method, Mark et al. [8], Alberto Zucchini et al. [9] computed stress intensity factors for thin cracked plates. Using complex variable method Zehnder et al. [10] calculated stress intensity factor for a finite crack in an infinite isotropic plate. The present method uses an integral transform technique and does not assume any symmetry about the co-ordinate axes. Also the constants appearing in the solution of the governing differential equations are obtained from the displacement boundary conditions by defining the derivative of the displacement discontinuities on the crack surfaces apart from the moment boundary conditions and continuity conditions. In the present study, the general formulae for the stress distribution in an infinite elastic plate containing cracks are derived and the stress intensity factor is determined in a closed form in the case of a single crack when the plate is subjected to out-of-plane moments and the results are compared with those from the literature.

\section{Formulation of the Problem}

Let us consider the cases of bending or twisting actions of an infinite plate by moments that are uniformly dis- tributed along the edges of the plate containing collinear cracks. We take $x y$-plane to coincide with the middle plane of the plate before deformation. The $z$-axis is assumed to be perpendicular to the middle plane. We denote the bending moment per unit length about $x$-axis by $M_{y y}$ and about y-axis by $M_{x x}$ and the twisting moment per unit length by $M_{x y}$. Let $Q_{x}$ and $Q_{y}$ be the shear forces components. The thickness of the plate is $h$ and we consider it to be small in comparison with other dimensions. Let us assume that during bending, the plate undergoes the displacement $w$ perpendicular to $x y$-plane. In the present analytical method, we consider the problem in which an infinite elastic plate, contains cracks located on a single line is acted upon by applied moments. Let the co-ordinate system be so chosen that the $x$-axis coincides with the line on which the cracks are located. Let $L$ denote the union of intervals occupied by the cracks on the $x$-axis and $M$ is the interval not occupied by the cracks. Suppose that a thin plate containing a crack is subjected to uniform bending or twisting moments at infinity. Since the crack surface is traction-free the boundary conditions along the crack surface permitting all of the free edge conditions for the present problem is given by the following equations:

The free boundary conditions on the crack surface are given by,

$$
\begin{gathered}
M_{x y}(x, 0)=0, x \in L \\
M_{y y}(x, 0)=0, x \in L \\
Q_{y}(x, 0)=0, x \in L
\end{gathered}
$$

The solution to this problem may be obtained by superposing the simple solution of an uncracked plate under uniform bending moment or twisting moment to that of a cracked plate with bending or twisting moment applied to the crack surfaces. That is, the solution may be obtained by using standard superposition technique and thus for the purpose of evaluating the crack tip singular stresses it is sufficient to consider the problem in which self-equilibrating crack surface loads are the only external loads. Thus, it suffices to solve the problem of specifying uniform bending and twisting moment on the crack segment of the plate. Let the desired system be composed of two parts, one the uniform moment field and the other a perturbation field due to the crack which dies out at infinity. While the boundary conditions along the free edges of the crack require traction free conditions, it is possible to formulate the problem as one of finding solutions for the perturbation solutions satisfying the field equations and the boundary conditions

$$
M_{x y}(x, 0)=\frac{G^{*}(x)}{2}, x \in L
$$




$$
\begin{gathered}
M_{y y}(x, 0)=\frac{H^{*}(x)}{2}, x \in L \\
Q_{y}(x, 0)=0
\end{gathered}
$$

The equilibrium equations are given by,

$$
\begin{gathered}
\frac{\partial M_{x y}}{\partial x}-\frac{\partial M_{y}}{\partial y}+Q_{y}=0 \\
\frac{\partial M_{y x}}{\partial y}+\frac{\partial M_{x}}{\partial x}+Q_{x}=0 \\
\frac{\partial Q_{x}}{\partial x}+\frac{\partial Q_{y}}{\partial y}=0
\end{gathered}
$$

Also, the stress components are the linear combination of the variable $z$.

$$
\sigma_{x y}=\frac{12 M_{x y} z}{h^{3}} ; \sigma_{y y}=\frac{12 M_{y y} z}{h^{3}} ; \sigma_{x x}=\frac{12 M_{x x} z}{h^{3}}
$$

The strain compatibility equation is given by,

$$
\frac{\partial^{2} \varepsilon_{x x}}{\partial y^{2}}+\frac{\partial^{2} \varepsilon_{y y}}{\partial x^{2}}=\frac{\partial^{2} \varepsilon_{x y}}{\partial x \partial y}
$$

If we define the moment resultants in terms of the biharmonic function $\varphi(x, y)$ as given by

$$
M_{x}=\frac{\partial^{2} \phi}{\partial y^{2}} ; M_{y}=\frac{\partial^{2} \phi}{\partial x^{2}} ; M_{x y}=\frac{\partial^{2} \phi}{\partial x \partial y}
$$

then the governing Equations (6)-(8) are satisfied. From the compatibility conditions (10), the present problem reduces to that of solving the bi-harmonic equation in $\phi(x, y)$

$$
\nabla^{4} \phi=0
$$

where, $\quad \nabla^{4} \phi=\frac{\partial^{4} \phi}{\partial x^{4}}+2 \frac{\partial^{4} \phi}{\partial x^{2} \partial y^{2}}+\frac{\partial^{4} \phi}{\partial y^{4}}$

Let $G^{(1)}(\xi, y)$ be the Fourier transform of $\phi(x, y)$ for $y>0$. Then

$$
G^{(1)}(\xi, y)=\int_{-\infty}^{\infty} \phi^{(1)}(x, y) \mathrm{e}^{i \xi x} \mathrm{~d} x, y>0
$$

Taking Fourier transformation of the bi-harmonic equation w. $r$. $t$ the variable $\mathrm{x}$, we get the ordinary differential equation in $G(\xi, y)$ as given by

$$
\left(\frac{\mathrm{d}^{2}}{\mathrm{~d} y^{2}}-\xi^{2}\right)^{2} G(\xi, y)=0
$$

whose solutions are given by

$$
\begin{aligned}
& G^{(1)}(\xi, y)=\left[P_{1}(\xi)+y Q_{1}(\xi)\right] \mathrm{e}^{-|\xi| y} \quad y>0 \\
& G^{(2)}(\xi, y)=\left[P_{2}(\xi)+y Q_{2}(\xi)\right] \mathrm{e}^{|\xi| y} \quad y<0
\end{aligned}
$$

where $G^{(2)}$ is the Fourier transformation of $\phi(x, y)$ for $y<0$, and $P_{1}(\xi), P_{2}(\xi), Q_{1}(\xi), Q_{2}(\xi)$ are the unknown functions to be determined. From the moment boundary conditions we have the following equations,

$$
\begin{aligned}
& M_{y y}^{(1)}(x, 0)-M_{y y}^{(2)}(x, 0)=0, \forall x \\
& M_{x y}^{(1)}(x, 0)-M_{x y}^{(2)}(x, 0)=0, \forall x
\end{aligned}
$$

The bending and twisting moments in terms of $G^{(1)}(\xi, y)$ for $y>0$ are given by

$$
\begin{gathered}
M_{x}^{(1)}(x, y)=\frac{1}{2 \pi} \int_{-\infty}^{\infty} \frac{\partial^{2} G^{(1)}(\xi, y)}{\partial y^{2}} \mathrm{e}^{-i \xi x} \mathrm{~d} \xi, y>0 \\
M_{y}^{(1)}(x, y)=\frac{1}{2 \pi} \int_{-\infty}^{\infty} \xi^{2} G^{(1)}(\xi, y) \mathrm{e}^{-i \xi x} \mathrm{~d} \xi, y>0 \\
M_{x y}^{(1)}(x, y)=-\frac{i}{2 \pi} \int_{-\infty}^{\infty} \xi \frac{\partial G^{(1)}(\xi, y)}{\partial y} \mathrm{e}^{-i \xi x} \mathrm{~d} \xi, y>0
\end{gathered}
$$

Similarly we get the bending and twisting moments for $y<0$ in terms of $G^{(2)}(\xi, y)$

The bending and twisting moments in terms of $G^{(2)}(\xi, y)$ for $y<0$ are given by

$$
\begin{aligned}
& M_{x}^{(2)}(x, y)=\frac{1}{2 \pi} \int_{-\infty}^{\infty} \frac{\partial^{2} G^{(2)}(\xi, y)}{\partial y^{2}} \mathrm{e}^{-i \xi x} \mathrm{~d} \xi, y<0 \\
& M_{y}^{(2)}(x, y)=\frac{1}{2 \pi} \int_{-\infty}^{\infty} \xi^{2} G^{(2)}(\xi, y) \mathrm{e}^{-i \xi x} \mathrm{~d} \xi, y<0 \\
& M_{x y}^{(2)}(x, y)=-\frac{i}{2 \pi} \int_{-\infty}^{\infty} \xi \frac{\partial G^{(2)}(\xi, y)}{\partial y} \mathrm{e}^{-i \xi x} \mathrm{~d} \xi, y<0
\end{aligned}
$$

The displacement components are given by the following expressions:

$$
\begin{aligned}
& u_{x}=-z \frac{\partial w}{\partial x} \\
& u_{y}=-z \frac{\partial w}{\partial y}
\end{aligned}
$$

The displacement boundary conditions are given by

$$
\begin{aligned}
& A(x)=0, x \in M \\
& B(x)=0, x \in M
\end{aligned}
$$

where the displacement discontinuities are defined by the functions $A(x), B(x)$

$$
\begin{aligned}
& A(x)=\frac{\partial}{\partial x}\left[u_{x}^{(1)}(x, 0)-u_{x}^{(2)}(x, 0)\right], x \in L \\
& B(x)=\frac{\partial}{\partial x}\left[u_{y}^{(1)}(x, 0)-u_{y}^{(1)}(x, 0)\right], x \in L
\end{aligned}
$$


and the superscripts (1) and (2) denote the components in the upper half plane $y>0$ and lower half plane $y<0$ respectively.

From the continuity conditions and the moment boundary conditions we get the four simultaneous equa-

$$
\begin{gathered}
\xi^{2}(1+v)\left(P_{1}-P\right)_{2}-2|\xi|\left(Q_{1}+Q_{2}\right)=-\bar{A}(\xi) D\left(1-v^{2}\right) \\
-i \xi|\xi|(1+v)\left(P_{1}+P\right)_{2}+i \xi\left(Q_{1}-Q_{2}\right)(3+v)=\bar{B}(\xi) D\left(1-v^{2}\right)
\end{gathered}
$$

tions for solving $P_{1}(\xi), P_{2}(\xi), Q_{1}(\xi), Q_{2}(\xi)$

$$
\begin{gathered}
P_{1}=P_{2} \\
Q_{1}-\xi P_{1}=Q_{2}+|\xi| P_{2}
\end{gathered}
$$

$$
\begin{gathered}
Q_{1}=\frac{1}{4 \xi}[\bar{A} \operatorname{sgn}(\xi)-i \bar{B}] D\left(1-v^{2}\right) \\
Q_{2}=\frac{1}{4 \xi}[\bar{A} \operatorname{sgn}(\xi)+i \bar{B}] D\left(1-v^{2}\right)
\end{gathered}
$$

$$
\begin{aligned}
& \bar{M}_{y}(\xi, y)=-\xi^{2} G(\xi, y) \\
& \bar{M}_{x x}(\xi, y)=\frac{\partial^{2} G}{\partial y^{2}} \\
& \bar{M}_{x y}(\xi, y)=i \xi \frac{\partial G}{\partial y}
\end{aligned}
$$

Substituting the values of $P_{1}(\xi), Q_{1}(\xi)$ into (21), we get the bending moment resultants in the upper half plane $y>0$, in the transformed co-ordinates as given by the following equation,

$$
M_{y}^{(1)}(x, y)=\frac{-D\left(1-v^{2}\right)}{8 \pi} \int_{-\infty}^{\infty}\{\bar{A} y|\xi|-\bar{B} i \operatorname{sgn}(\xi)(1+|\xi| y)\} \mathrm{e}^{-i \xi x-|\xi| y} \mathrm{~d} \xi, y>0
$$

Performing the inner integral in terms of $A(s)$ and $B(s)$ we get the bending moment resultants in the upper half-plane $y>0$, in terms of the unknown displacement functions $A(s)$ and $B(s)$ as given by

$$
M_{y}^{(1)}(x, y)=\frac{-D(1-v)^{2}}{4 \pi}\left[\int_{-\infty}^{\infty} A(s) y\left\{\frac{\left[y^{2}-(x-s)^{2}\right]}{\left[(x-s)^{2}+y^{2}\right]} \mathrm{d} s\right\}+\int_{-\infty}^{\infty} B(s)(x-s)\left\{\frac{\left[(x-s)^{2}+3 y^{2}\right]}{\left[(x-s)^{2}+y^{2}\right]^{2}} \mathrm{~d} s\right\}\right] y>0
$$

Substituting the values of $P_{2}(\xi), Q_{2}(\xi)$ into (24), we get the bending moment resultants in the lower half plane

$y<0$, in the transformed coordinates as given by the following equation

$$
M_{y}^{(2)}(x, y)=\frac{-D\left(1-v^{2}\right)}{8 \pi} \int_{-\infty}^{\infty}\{\bar{A} y|\xi|-\bar{B} i \operatorname{sgn}(\xi)(1-|\xi| y)\} \mathrm{e}^{-i \xi x+|\xi| y} \mathrm{~d} \xi, y<0
$$

Performing the inner integral in terms of $A(s)$ and $B(s)$ we get the bending moment resultants in the lower

half-plane $y<0$, in terms of the unknown displacement functions $A(s)$ and $B(s)$ as given by

$$
M_{y}^{(2)}(x, y)=\frac{-D\left(1-v^{2}\right)}{4 \pi}\left[\int_{-\infty}^{\infty} A(s) y\left\{\frac{\left[y^{2}-(x-s)^{2}\right]}{\left[\left({ }^{2}-s\right)^{2}+y^{2}\right]} \mathrm{d} s\right\}+\int_{-\infty}^{\infty} B(s)(x-s)\left\{\frac{\left[(x-s)^{2}+3 y^{2}\right]}{\left[(x-s)^{2}+y^{2}\right]^{2}} \mathrm{~d} s\right\}\right] y<0,
$$

Combining the Equations (35b) and (35d) we get the

expression for bending moment resultant,

$$
M_{y}(x, y)=\frac{-D\left(1-v^{2}\right)}{4 \pi}\left[\int_{-\infty}^{\infty} A(s) y\left\{\frac{\left[y^{2}-(x-s)^{2}\right]}{\left[(x-s)^{2}+y^{2}\right]} \mathrm{d} s\right\}+\int_{-\infty}^{\infty} B(s)(x-s)\left\{\frac{\left[(x-s)^{2}+3 y^{2}\right]}{\left[(x-s)^{2}+y^{2}\right]^{2}} \mathrm{~d} s\right\} y \neq 0,\right.
$$


Similarly the expression for the twisting moment $M_{x y}(x, y)$ as given by

$$
M_{x y}(x, y)=\frac{-D\left(1-v^{2}\right)}{4 \pi}\left[\int_{-\infty}^{\infty} A(s)(x-s)\left\{\frac{\left[(x-s)^{2}-y^{2}\right]}{\left[(x-s)^{2}+y^{2}\right]^{2}} \mathrm{~d} s\right\}+\int_{-\infty}^{\infty} B(s) y\left\{\frac{\left[3(x-s)^{2}+y^{2}\right]}{\left[(x-s)^{2}+y^{2}\right]^{2}} \mathrm{~d} s\right\} y \neq 0,\right.
$$

where $A(s)$ and $B(s)$ are the unknown functions to be determined from the given boundary conditions. The limiting values as $y \rightarrow 0+$ and $y \rightarrow 0$ - of the bending and twisting moments along the crack line are given by,

$$
\begin{gathered}
M_{y}(x, 0)=\frac{-D\left(1-v^{2}\right)}{4 \pi} \int_{-\infty}^{\infty} \frac{B(s)}{x-s} \mathrm{~d} s \\
M_{x y}(x, 0)=\frac{-D\left(1-v^{2}\right)}{4 \pi} \int_{-\infty}^{\infty} \frac{A(s)}{x-s} \mathrm{~d} s
\end{gathered}
$$

By using the conditions (4)-(5) in the above expressions, the interval of integration reduces to $L$. From the boundary conditions (4) and the above relations we get the singular integral equations

$$
\begin{aligned}
& \int_{-L}^{L} \frac{A(s)}{x-s} \mathrm{~d} s=\left(\frac{1}{\alpha}\right) G^{*}(x), x \in L \\
& \int_{-L}^{L} \frac{B(s)}{x-s} \mathrm{~d} s=\left(\frac{1}{\alpha}\right) H^{*}(x), x \in L
\end{aligned}
$$

where $\alpha=\frac{-D\left(1-v^{2}\right)}{2 \pi}$, for the determination of unknown functions $A$ and $B$ on the interval $L$. Once the functions $A(s)$ and $B(s), \quad x \in L$ are known, the bending and twisting moments for the crack problem are determined.

\section{Single Crack Problem}

In order to illustrate the present procedure, we give the details in the case of a single crack opened by the action of symmetric bending load applied at the edges of the plate. In this section, we consider the problem of determining the distribution of bending stress in the vicinity of a Griffith crack of length $2 c$, occupying the interval ($c, c)$ on the $\mathrm{x}$-axis in an infinite isotropic elastic plate. The bending moment resultants and the transverse shear force components are given by,

$$
\begin{aligned}
& M_{x}=-D\left[\frac{\partial^{2} w}{\partial x^{2}}+v \frac{\partial^{2} w}{\partial y^{2}}\right]+\frac{h^{2}}{5} \frac{\partial Q_{x}}{\partial x} \\
& M_{y}=-D\left[\frac{\partial^{2} w}{\partial y^{2}}+v \frac{\partial^{2} w}{\partial x^{2}}\right]+\frac{h^{2}}{5} \frac{\partial Q_{y}}{\partial y}
\end{aligned}
$$

$$
\begin{aligned}
& Q_{x}=-D \frac{\partial}{\partial x}\left[\frac{\partial^{2} w}{\partial x^{2}}+\frac{\partial^{2} w}{\partial y^{2}}\right]+\frac{1}{(1-v)} \frac{\partial \psi}{\partial y} \\
& Q_{y}=-D \frac{\partial}{\partial y}\left[\frac{\partial^{2} w}{\partial x^{2}}+\frac{\partial^{2} w}{\partial y^{2}}\right]-\frac{1}{(1-v)} \frac{\partial \psi}{\partial x}
\end{aligned}
$$

Taking Fourier transform of the above equations w. r. t. $x$, we get the displacement component in terms of the bending moment components in the transformed co-ordinate system as given by,

$$
\left[-\xi^{2} \bar{w}+\frac{\partial^{2} \bar{w}}{\partial y^{2}}\right]=\frac{-\left(\bar{M}_{x}+\bar{M}_{y}\right)}{D(1+v)}
$$

The transverse shear force $\bar{Q}_{y}$ are given by,

$$
\bar{Q}_{y}=\frac{\partial}{\partial y}\left[\frac{\bar{M}_{x}+\bar{M}_{y}}{(1+v)}\right]-i \xi \bar{\psi}
$$

where $\psi$ is calculated from the equation,

$$
\frac{\partial}{\partial y}\left(\Delta \psi-\frac{10}{h^{2}} \psi\right)=0
$$

Taking Fourier Transform of the above Equation (47) we get

$$
\begin{gathered}
\frac{\partial}{\partial y}\left[-\left(\xi^{2}+\lambda^{2}\right) \bar{\psi}+\frac{d^{2} \bar{\psi}}{d y^{2}}\right]=0 \\
\bar{\psi}=c_{1} \sqrt{\xi^{2}+\lambda^{2}} \mathrm{e}^{-y \sqrt{\xi^{2}+\lambda^{2}}}+c_{2} \sqrt{\xi^{2}+\lambda^{2}} \mathrm{e}^{y \sqrt{\xi^{2}+\lambda^{2}}}
\end{gathered}
$$

where $\lambda=\frac{\sqrt{10}}{h}$

Since $Q_{x} \rightarrow 0$ and $Q_{y} \rightarrow 0$ as $y \rightarrow \infty$ we have $c_{2}=0$

$$
\bar{\psi}=c_{1} \sqrt{\xi^{2}+\lambda^{2}} \mathrm{e}^{-y \sqrt{\xi^{2}+\lambda^{2}}}
$$

The constant 0 is determined from

$$
\begin{aligned}
& Q_{y}(x, 0)=0,|x| \leq c \\
& \bar{Q}_{y}=\frac{\partial}{\partial y}\left[\frac{\bar{M}_{x}+\bar{M}_{y}}{(1+v)}\right]-i \xi \bar{\psi}
\end{aligned}
$$

Substituting $\bar{M}_{x}(\xi, y), \bar{M}_{y}(\xi, y)$ and $\bar{\psi}(\xi, y)$ in (46) and using the crack surface boundary condition,

$$
Q_{y}(x, 0)=0,|x| \leq c
$$


the constant is given by,

$$
\begin{gathered}
C_{1}=\frac{D(1-v)^{2}}{2 \pi} \bar{B} \\
\psi(x, y)=\frac{D(1-v)^{2}}{8 \pi} \int_{-c}^{c} B(s)\left\{\frac{\lambda y}{\sqrt{(x-s)^{2}+y^{2}}} K_{1}\left(\lambda \sqrt{(x-s)^{2}+y^{2}}\right)\right\} \mathrm{d} s \\
\frac{h^{2}}{5}\left[\frac{\partial^{2} \psi(x, y)}{\partial x \partial y}\right]_{y=0}=\frac{D(1-v)^{2}}{8 \pi} \int_{-c}^{c} B(s) \frac{h^{2}}{5} \lambda^{2}\left[\frac{\partial}{\partial x}\left[\lambda \frac{K_{1}\left(\lambda \sqrt{x-s^{2}+y^{2}}\right)}{\lambda \sqrt{x-s^{2}+y^{2}}} \int_{-c}^{c} B(s)\left[\int_{-\infty}^{\infty} \mathrm{e}^{-y \sqrt{\xi^{2}+\lambda^{2}}}\right]_{y=0}\right]\right. \\
\frac{h^{2}}{5}\left[\frac{\partial^{2} \psi(x, y)}{\partial x \partial y}\right]_{y=0}=\frac{-D(1-v)^{2}}{8 \pi} \int_{-c}^{c} \frac{B(s)}{x-s} \mathrm{~d} s \frac{h^{2}}{5} \lambda^{2} K_{2}[\lambda x] \\
L t \quad \frac{h^{2}}{5}\left[\frac{\partial^{2} \psi(x, y)}{\partial x \partial y}\right]_{y=0}=\frac{-D(1-v)^{2}}{8 \pi}\left[\int_{-c}^{c} \frac{B(s)}{x-s} \mathrm{~d} s \frac{L t}{h \rightarrow 0} \frac{h^{2}}{5} \lambda^{2}\left\{-\frac{1}{2}+\frac{2}{\lambda^{2} x^{2}}\right\}\right] \\
L t \quad \frac{h^{2}}{5}\left[\frac{\partial^{2} \psi(x, y)}{\partial x \partial y}\right]_{y=0}=\frac{D(1-v)^{2}}{8 \pi} \int_{-c}^{c} \frac{B(s)}{x-s} \mathrm{~d} s
\end{gathered}
$$

From (50), (52) the function $\psi(x, y)$ is given by,

The bending moment $M_{y}(x, 0)$ along the crack line $y=0$, as $h \rightarrow 0$ is given by

$$
M_{y}(x, 0)=\frac{-D\left(1-v^{2}\right)}{4 \pi} \int_{-c}^{c} \frac{B(s)}{x-s} \mathrm{~d} s-\frac{D(1-v)^{2}}{8 \pi} \int_{-c}^{c} \frac{B(s)}{(x-s)} \mathrm{d} s
$$

Making use of the boundary condition (4) we get the singular integral equation for the determination of $\mathrm{B}(\mathrm{s})$ as given by,

$$
\begin{aligned}
B(s)= & -\frac{(1+v)}{(3+v)} \frac{1}{D\left(1-v^{2}\right)} \frac{4}{\pi \sqrt{a^{2}-s^{2}}} \\
& \int_{-c}^{c} \frac{H^{*}(x) \sqrt{\left(c^{2}-t^{2}\right)}}{x-s} \mathrm{~d} x
\end{aligned}
$$

$\frac{D\left(1-v^{2}\right)}{8 \pi} \frac{(3+v)}{(1+v)} \int_{-c}^{c} \frac{B(s)}{(x-s)} \mathrm{d} s=\frac{H^{*}(x)}{2}|x| \leq c$

Solving for $\mathrm{B}(\mathrm{s})$, we get the following expression for $\mathrm{B}(\mathrm{s})$,

$$
\begin{aligned}
& M_{y}(x, 0)=\frac{\operatorname{sgn}(x)}{2 \pi \sqrt{\left(x^{2}-c^{2}\right)}} \frac{(1+v)}{(3+v)} \int_{-c}^{c} \frac{H^{*}(x) \sqrt{\left(c^{2}-t^{2}\right)}}{t-x} \mathrm{~d} t \\
& H^{*}(x) / 2=M_{0}
\end{aligned}
$$

The bending moment resultant along the crack line from the above equation is given by,

$$
M_{y}(x, 0)=\frac{\operatorname{sgn}(x) x}{\sqrt{\left(x^{2}-c^{2}\right)}} \frac{(1+v)}{(3+v)} M_{0}
$$

The bending stress $\sigma_{y}(x, 0)$ along the crack line

$$
\begin{aligned}
y=0, \text { as } h & \rightarrow 0 \text { is given by } \\
\sigma_{y y}(x, 0) & =\frac{12 M_{0} z \operatorname{sgn}(x) x}{h^{3} \sqrt{\left(x^{2}-c^{2}\right)}} \frac{(1+v)}{(3+v)},|x| \geq c
\end{aligned}
$$

The bending stress intensity factor $K_{I}$ due to bending moment at $\mathrm{z}=\mathrm{h} / 2$ is given by

$$
K_{I}=\operatorname{Lim}_{x \rightarrow c}\left\{\sqrt{[2(x-c)]} \sigma_{y y}(x, 0)\right\}=\frac{6 M_{0} \sqrt{c}}{h^{2}} \frac{(1+v)}{(3+v)}
$$


For small $\left|\frac{h}{a}\right|$, the bending moment on the crack line $\mathrm{y}=0$ is calculated as follows:

$$
\frac{h^{2}}{5}\left[\frac{\partial^{2} \psi(x, y)}{\partial x \partial y}\right]_{y=0}=\frac{-D(1-v)^{2}}{8 \pi} K_{2}[\lambda x] \int_{-c}^{c} \frac{B(s)}{x-s} \mathrm{~d} s
$$

where

$$
K_{2}(\lambda x)=-\left\{\ln \left(\frac{\lambda x}{2}\right)+\gamma-\frac{1}{\lambda^{2} x^{2}}\right\} ; \lambda=\frac{\sqrt{10}}{h} ; \gamma=0.5772
$$

Hence the bending moment along the crack line $y=0$ is given by,

$$
M_{y}(x, 0)=\frac{-D\left(1-v^{2}\right)}{4 \pi} \int_{-\infty}^{\infty} \frac{B(s)}{x-s} \mathrm{~d} s-\frac{D(1-v)^{2}}{8 \pi}\left\{\ln \left(\frac{\lambda x}{2}\right)+\gamma-\frac{1}{\lambda^{2} x^{2}}\right\} \int_{-c}^{c} \frac{B(s)}{x-s} \mathrm{~d} s
$$

Substituting the value of $\mathrm{B}(\mathrm{s})$ from (61) into the above bending moment along the crack line $\mathrm{y}=0$ as given by, equation and performing the inner integral we get the

$$
M_{y}(x, 0)=\frac{\operatorname{sgn}(x) x M_{0}}{\sqrt{\left(x^{2}-c^{2}\right)}}\left[2 \frac{(1+v)}{(3+v)}-\frac{\left(1-v^{2}\right)}{4(1+v)(3+v)}\left\{\ln (\lambda x)+\gamma-\frac{1}{\lambda^{2} x^{2}}\right\}\right],|x| \geq c
$$

The bending stress $\sigma_{y}(x, 0)$ along the crack line $\quad y=0$, is given by

$$
\sigma_{y}(x, 0)=\frac{12 \operatorname{sgn}(x) x z M_{0}}{h^{3} \sqrt{\left(x^{2}-c^{2}\right)}}\left[2 \frac{(1+v)}{(3+v)}-\frac{\left(1-v^{2}\right)}{4(1+v)(3+v)}\left\{\ln (\lambda x)+\gamma-\frac{1}{\lambda^{2} x^{2}}\right\},|x| \geq c\right.
$$

Stress intensity factor $K_{I}$ at $\mathrm{z}=\mathrm{h} / 2$, due to bending mo- ment is given by

$$
K_{I}=\lim _{x \rightarrow c}\left\{\sqrt{[2(x-c)]} \sigma_{y y}(x, 0)\right\}=\frac{6 M_{0} \sqrt{c}}{h^{2}}\left[2 \frac{(1+v)}{(3+v)}-\frac{\left(1-v^{2}\right)}{4(1+v)(3+v)}\left\{\ln (\lambda c)+\gamma-\frac{1}{\lambda^{2} c^{2}}\right\}\right]
$$

The graph of non-dimensional stress intensity factor vs. thickness for $v=0.3$ is plotted in Figure 2 and the stress intensity factor is in good agreement with the results in [2] and [3]. The stress variation near the crack tip, calculated from Equation (69) is plotted in Figure 3. For example, a value of $c / h=5.0$ is assumed for calculation.

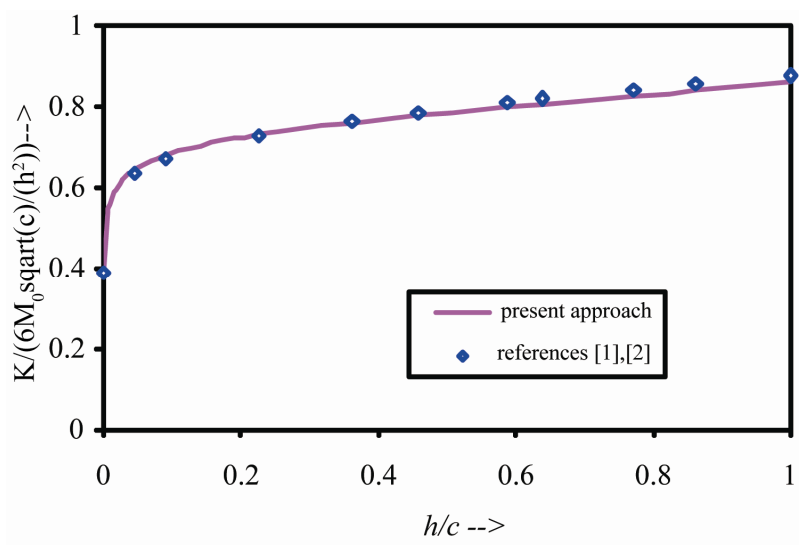

Figure 2. Variation of non-dimensional stress intensity with thickness of the plate.

\section{Results and Discussion}

The variation of non-dimensional stress intensity stress intensity factor with thickness of the plate is shown in Figure 2. The small differences between the present results and in the references [2] and [3] may be due to two

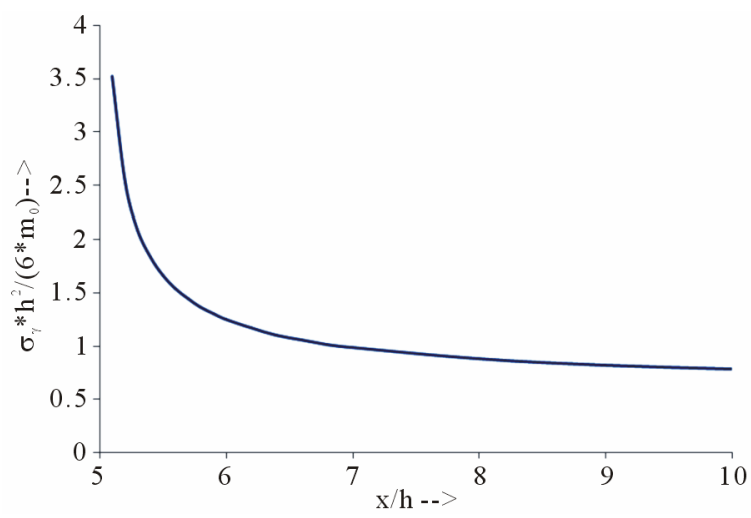

Figure 3. Stress $\left(\sigma_{y}(x, 0)\right)$ distribution near the crack tip for $|x| \geq c, \mathbf{c} / \mathbf{h}=\mathbf{5 . 0}$. 
different approaches being used in [2] and [3]. Hartranft and Sih [2] used more rigorous method using eigenfunction expansions for plate bending problem introducing the effect of plate thickness on crack-tip stress distribution. The approximate method based partly on finite element analysis and partly on a continuum analysis using Irwin's [11] solution for an infinite plate is used in [3]. Figure 3 shows the exponential variation of normal stress component near the crack tip for $|x| \geq c, z=h / 2$. It decreases away from the crack tip as expected. Future work in this direction is planned to solve composite plate problems with delamination.

\section{Conclusions}

A simple method for determining the analytical expression for the bending stress distribution, in the vicinity of a crack in an infinite elastic plate using Reissner plate theory is explained. The general formulae for the bending moment and twisting moment in an elastic plate containing cracks located on a single line are derived. The solution is obtained in a closed form for the case in which there is a single crack in an infinite plate and the stress intensity factor is calculated as a function of plate thickness, when the plate is subjected to symmetric bending loads. The stress intensity factor is compared with that obtained in the literature.

\section{References}

[1] E. Reissner, "The Effect of Transverse Shear Deformation on the Bending of Elastic Plates," ASME Journal of Applied Mechanics, Vol. 12, 1945, pp. A68-A77.

[2] R. J. Hartranft and G. C. Sih, "Effect of Plate Thickness on the Bending Stress Distribution around Through Cracks," Journal of Mathematics and Physics, Vol. 47, 276-291,1968
[3] S. Viswanath, "On the Bending of Plates with through Cracks from Higher Order Plate Theories," Ph. D Thesis, Indian Institute of Science, 1985.

[4] M. L. Williams, "The Bending Stress Distribution at the Base of a Stationary Crack," ASME Journal of Applied Mechanics, Vol. 28, 1961, pp. 78-82.

[5] G. C. Sih, P. C. Paris and F. Erdogan, "Crack-Tip, Stress-Intensity Factors for Plate Extension and Plate Bending Problems," ASME Journal of Applied Mechanics, Vol. 9, 1962, pp. 306-312.

[6] S. Krenk, "The Stress Distribution in an Infinite Anisotropic Plate with Collinear Cracks," International Journal of Solids and Structures, Vol. 11, No. 4, 1975, pp. 449-460. doi:10.1016/0020-7683(75)90080-3

[7] R. S. Alwar and K. N. Ramachandran, "Influence of Crack Closure on the Stress Intensity Factor for Plates Subjected to Bending-A 3-D Finite Element Analysis," Engineering Fracture Mechanics, Vol. 17, No. 4, 1983, pp. 323-333. doi:10.1016/0013-7944(83)90083-8

[8] M. J. Viz, D. O. Potyondy, A. T. Zehnder, C. C. Rankin and E. Riks, "Computation of Membrane and Bending Stress Intensity Factors for Thin, Cracked Plates," International Journal of Fracture, Vol. 72, No. 1, 1995, pp. 21-38. doi:10.1007/BF00036927

[9] A. Zucchini, C.-Y. Hui and A. T. Zehnder, "Crack Tip Stress Fields for Thin Plates in Bending, Shear and Twisting: A Three Dimensional Finite Element Study," International Journal of Fracture, Vol. 104, No. 4, 2000, pp. 387-407.

[10] A. T. Zehnder and C.-Y. Hui, "Stress Intensity Factors for Plate Bending and Shearing Problems," Journal of Applied Mechanics, Vol. 61, No. 3, 1994, pp. 719-722. doi:10.1115/1.2901522

[11] G. R. Irwin, "Analysis of Stresses and Strains near the End of a crack Traversing a Plate," Journal of applied Mechanics, 1957, Vol. 24, pp. 361-364. 